\title{
Flight Mechanics of a Tail-less Articulated Wing Aircraft
}

\author{
Aditya A. Paranjape* and Soon-Jo Chung ${ }^{\dagger}$
}

\begin{abstract}
This paper explores the flight mechanics of a Micro Aerial Vehicle (MAV) without a vertical tail. The key to stability and control of such an aircraft lies in the ability to control the twist and dihedral angles of both wings independently. Specifically, asymmetric dihedral can be used to control yaw whereas antisymmetric twist can be used to control roll. It has been demonstrated that wing dihedral angles can regulate sideslip and speed during a turn maneuver. The role of wing dihedral in the aircraft's longitudinal performance has been explored. It has been shown that dihedral angle can be varied symmetrically to achieve limited control over aircraft speed even as the angle of attack and flight path angle are varied. A rapid descent and perching maneuver has been used to illustrate the longitudinal agility of the aircraft. This paper lays part of the foundation for the design and stability analysis of an agile flapping wing aircraft capable of performing rapid maneuvers while gliding in a constrained environment.
\end{abstract}

\section{Nomenclature}

$\begin{array}{cl}D, L, Y & \text { drag, lift and side force } \\ J_{R, R}, J_{L, R} & \text { moment of inertia tensor of the right and left wings respectively, } \\ & \text { in their respective wing root frames } \\ J_{R}, J_{L}, J & \text { moment of inertia tensor of the right and left wings, and the aircraft } \\ & \text { body respectively, in the aircraft body frame } \\ m_{w, R}, m_{w, L} & \text { mass of the right and left wings, respectively } \\ m & \text { total mass of the aircraft } \\ p, q, r & \text { body axis roll, pitch and yaw rates } \\ \mathbf{r}_{C G} & \text { position vector of the aircraft center of gravity } \\ u, v, w & \text { body axis aircraft wind velocity components } \\ \alpha & \text { angle of attack } \\ \beta & \text { sideslip angle (but see next entry) } \\ \beta_{\{\cdot\}} & \text { backward sweep on a wing (see subscripts) } \\ \gamma & \text { flight path angle } \\ \delta_{\{\cdot\}} & \text { actuator deflection angle (see subscripts) } \\ \phi, \theta, \psi & \text { Euler angles } \\ \theta_{\{\cdot\}} & \text { wing twist } \\ \omega_{\{\cdot\}} & \text { angular velocity (see subscripts) } \\ \text { Subscripts } & \text { B } \\ \mathrm{R} & \text { aircraft body } \\ \mathrm{L} & \text { horizontal tail deflection wing } \\ & \text { right wing }\end{array}$

*Graduate student, Aerospace Engineering, Univ. of Illinois at Urbana-Champaign (UIUC), Urbana, IL. Student member, AIAA. Email: paranja2@illinois.edu

†Assistant professor, Aerospace Engineering, UIUC, Urbana, IL. Senior member, AIAA. Email: sjchung@illinois.edu 


\section{Introduction}

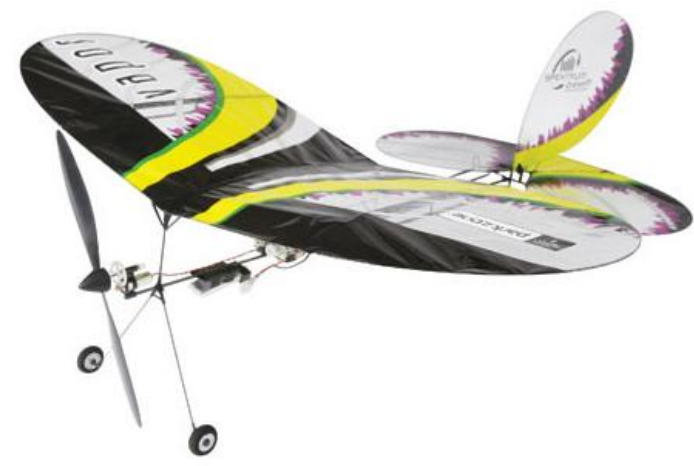

(a) ParkZone Vapor: the aircraft model considered in the paper (modelled without the vertical tail).

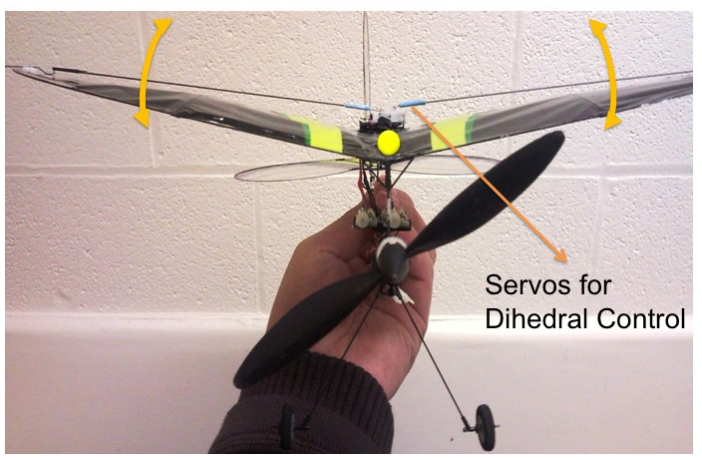

(b) Mechanism to control the wing dihedral.

Figure 1. Aircraft model considered in the paper, and a mechanism to control wing dihedral using wing flexibility in bending.

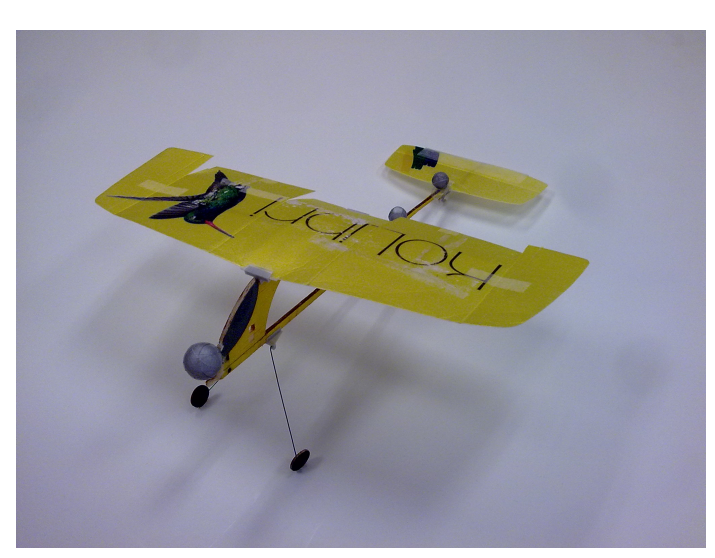

(a) The aircraft with wing dihedral set to zero.

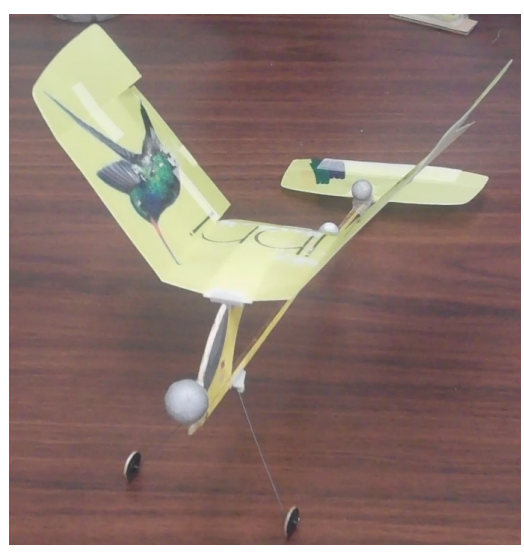

(b) The aircraft with its wings raised to a dihedral of $55 \mathrm{deg}$.

Figure 2. The Plantraco Kolibri Pocket Plane, used for experiements described in the paper.

There is a substantial interest in the aerospace community to learn and mimic avian flight. The challenge lies in developing unmanned aerial vehicles which have, among other attributes, (a) autonomy, (b) agility and (c) the ability to fly in constrained environments [1]. Birds are ubiquitous examples of such flyers and therefore, a natural choice as role models for miniature and micro aerial vehicles (MAVs) wherein these attributes can be engineered. Complex maneuvers require a combination of open and closed loop capabilities. However, the performance achievable in the closed loop (with control and guidance) is contingent upon the limitations of the airframe. The focus of this paper is on the airframe (open loop) rather than control. To simplify the analysis, the contributions of the fuselage and the thrust have been ignored with the understanding that they can be readily added within the conceptual framework of this paper.

In particular, this paper explores a new fixed wing aircraft concept motivated by avian wings. This paper considers a rigid wing which can be rotated at the root to generate variable dihedral. The dihedral angles on the port and starboard wings need not be equal. This concept of asymmetric dihedral is used to explore yaw 


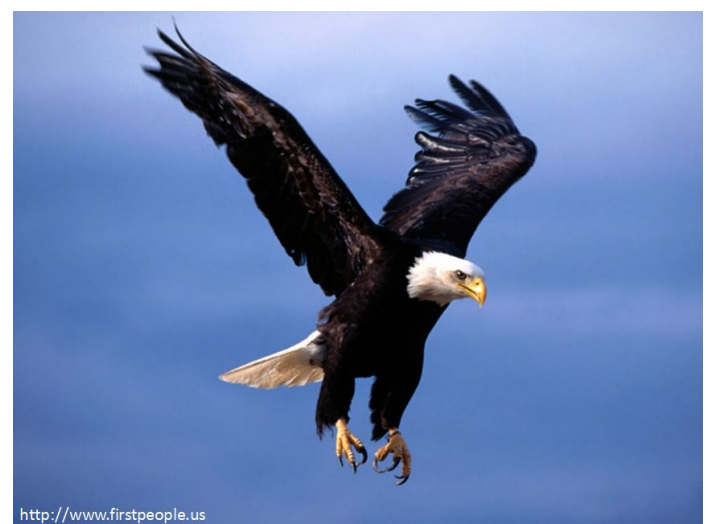

(a) Eagle in descent (source: firstpeople.us)

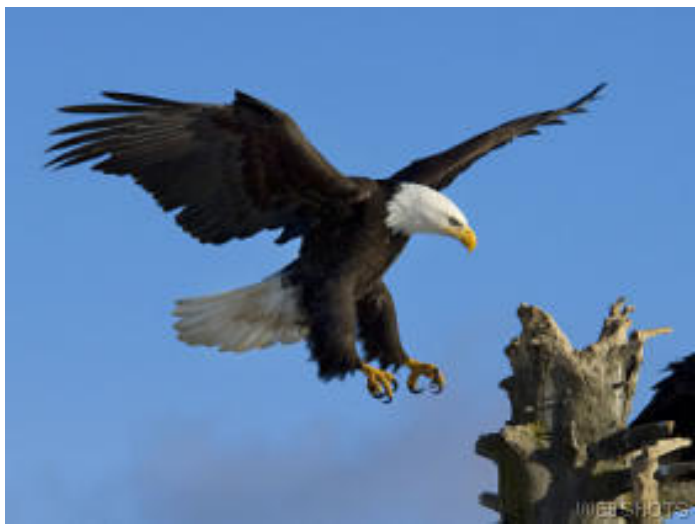

(b) Eagle while perching (source:yimwhan.com)

Figure 3. Eagle during a (steep) descent and during perching.

stability and control in the absence of a vertical tail. The aircraft model considered here is a modified version of the ParkZone Vapor, shown in Figs. 1(a) and 1(b). Figure 1(b) shows the mechanism being designed by the authors' research group to experimentally study the ideas presented in the present paper and in another upcoming paper for an aircraft with flexible wings [2]. The experiments described in this paper have been performed on a much smaller and lighter aircraft, called the Plantraco Kolibri Pocket Plane, shown in Fig. 2(a). Figure 2(b) shows the same aircraft with the dihedral angle of both wings raised to 55 deg.

\section{A. Literature Review}

Fixed and flapping wing MAVs have been extensively studied in literature. The compendium of papers in Ref. [3] is an excellent reference for some of the work done in this area until the turn of the $21^{\text {st }}$ century. More recently, Costello and Webb [4] demonstrated that articulated wing MAVs, with wings hinged at the root, experience reduced gust sensitivity. Wickenheiser and Garcia $[5,6]$ studied the dynamics of morphing aircraft and demonstrated perching using, among other forms of articulation, variable wing incidence. Reich et al [7] experimentally studied the aerodynamic performance of a wing of variable incidence for perching. The lateral stability and control of birds, and in particular, the role of wing dihedral, have been studied extensively by Sachs and co-authors [8-10]. Sachs has demonstrated that for air vehicles whose size and speed (and hence, the Reynolds number) are similar to that of birds, wings are sufficient to provide lateral stability thereby reducing, if not eliminating altogether, the need for a vertical tail. The lateral stability of tailless aircraft has been investigated by the authors in Ref. [11].

\section{B. Main Contributions and Outlook}

This paper is meant to contribute towards the broader problem of developing a flapping MAV capable of agile flight in constrained environments. Chung and Dorothy ${ }^{12}$ studied a neurobiologically-inspired controller for flapping flight, and demonstrated it on a robotic testbed. Their controller could switch in a stable and smooth fashion between flapping and gliding flight. Among other phases of flight, gliding is essential during landing. In particular, perching, inspired by bird flight, is viewed as an effective landing maneuver. Figure I illustrates an eagle as it descends and perches. Note the large dihedral during descent, and the nearly zero dihedral angle during perching. Perching, instead, requires substantial wing twist. This paper provides the flight mechanic foundations needed to design effective gliding and landing strategies.

The objective of this paper is to study the performance and stability of a vertical-tailless aircraft equipped with moving horizontal tail and wings. The twist and dihedral angles of both wings can be controlled independently. The idea is to employ antisymmetric wing twist to control roll and asymmetric dihedral to control yaw. Following Sachs and Holzapfel [8], the role of wing dihedral in controlling the longitudinal performance has been explored further. The aerodynamic forces on the aircraft wings and the horizontal tail 
are modelled using strip theory with aerodynamic data from Dickinson, Lehmann and Sane [13]. Bifurcation analysis [14] is used to study the performance and stability of a six degree-of-freedom aircraft model which incorporates the effect of dynamic CG location as a function of the aircraft geometry. Performance metrics of interest are (a) trim angle of attack, velocity, and flight path angle for longitudinal flight assessment, and (b) sideslip angle and turn rate for the lateral motion. Co-ordinated turns are analysed using a modified version of the constrained bifurcation analysis [14].

It is shown that large dihedral angles can be used for slow, steep descents at large angle of attack. The roll rate is observed to be highly sensitive to the wing twist. Relatively large dihedral angles are needed to achieve co-ordinated flight, which can be circumvented by appropriate centre of gravity (CG) placement. A perching-like maneuver has been demonstrated through simulation.

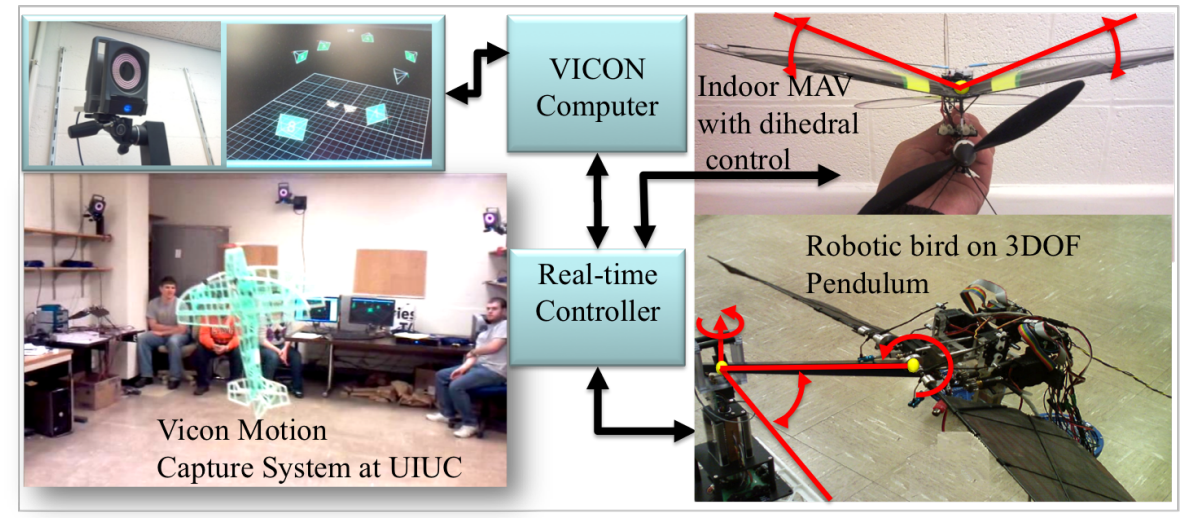

Figure 4. The VICON motion capture system, and its role in analysing and controlling indoor flight.

The ideas presented in this paper are being validated experimentally. Some results obtained during open loop studies have been presented in the paper. All positions were measured directly using a VICON motion capture system consisting of eight $2 \mathrm{MP}$ cameras. Figures 2(a) and 2(b) also show the reflective markers attached to the aircraft. These are used by VICON to locate the aircraft and measure its attitude. The VICON system and its role have been illustrated in Fig. 4. Efforts are under way to perform experiments with the aircraft flying in the closed loop.

The paper is organized as follows. The mathematical preliminaries are summarized in Section 2. Section 3 derives the dynamical equations and describes the aircraft model used in the subsequent analysis. In Section 4, an analytical comparison of differential dihedral and vertical tail vis-a-vis their role as yaw control devices is provided. A bifurcation analysis of the aircraft defined in the previous section is performed, with the objective of identifying the symmetric as well as asymmetric equilibria and their stability. Section 5 presents a simulated perching maneuver which demonstrates the agility of the aircraft. Section 6 describes the experimental results. Section 7 concludes the paper.

\section{Preliminaries}

In this section, trim computation and bifurcation analysis, required in the subsequent analysis, are briefly introduced. Consider a set of ordinary differential equations $\dot{x}=f(x, u, \lambda)$, where $x \in \mathbb{R}^{n}$ are the states and $u \in \mathbb{R}$ is the control input, and $\lambda \in \mathbb{R}^{p}$ are some system parameters. A trim $\left(x^{*}, u^{*}\right)$ is an equilbium point of this differential equation, i.e., $f\left(x^{*}, u^{*}\right)=0$. Given a control $u^{*}$, there may exist several trim states $\left(x^{*}, u^{*}\right)$. All of these trim states may not be of interest. Once a trim state $\left(x_{0}, u_{0}\right)$ is computed, $u_{0}$ is perturbed so that $u_{0} \mapsto u_{0}+\delta$, and $x_{0}$ is used as an initial estimate for the next trim $\left(x^{*}, u_{0}+\delta\right)$. The step size $\delta$ has to be chosen suitably. This is very similar to a procedure called numerical continuation. ${ }^{15}$

The Jacobian $\frac{\partial f}{\partial x}$ is computed at every trim state, and its eigenvalues are used to conclude the stability of the trim point. A trim point at which a solution branch undergoes a change in stability is called a bifurcation point. Bifurcations are important not only because they indicate the onset of instability, but also because trim branches or limit cycles originating from the bifurcation point provide indications about the behaviour of the aircraft after it has departed from the unstable trim state. One such bifurcation point is the saddle 
node bifurcation at which a solution branch turns around in the state space, leading to jumps. ${ }^{14}$ Another commonly encountered bifurcation is the Hopf bifurcation which gives rise to limit cycles (or oscillatory behaviour).

For this paper, the function fsolve in MATLAB ${ }^{\mathrm{TM}}$ has been used iteratively to compute trim states. In order to enable the code to handle folding solution branches, the equations of motion are augmented using the pseudo-arc-length ${ }^{15}$ Bifurcation points have not been computed explicitly. Instead, plots are showing trim state variables of interest versus the corresponding trim control variable carry a stability-based legend which allows bifurcation points to be identified along with their type.

\section{Equations of Motion}

\section{A. Frames of Reference}

Given frames $F$ and $G$, the matrix $T_{\mathrm{FG}}$ is a rotation matrix which transforms the components of a vector from the $G$ frame to $F$. The body frame, denoted by $B$, is attached to the body with the $x-z$ plane coincident with the aircraft plane of symmetry when the wings are undeflected. The $x$ axis points towards the aircraft nose. The $z$ axis points downwards, and the $y$ axis (pointing along the right undeflected wing) is defined to create a right handed coordinate system.

The frame $R$ is the frame based at the right wing root. Its $x$ axis coincides with the $x$ axis of the $B$ frame and it is related to the $B$ frame via a dihedral rotation $\delta_{R}$ at the wing root followed by a rotation $\theta_{R}$ about the $y$ axis. The $y$ axis points along the wing elastic axis. Let $R_{1}, R_{2}, R_{3}$ denote the body - wing frame rotation matrices for wing rotations about the root hinge corresponding to sweep $\left(\beta_{\{\cdot\}}\right)$, dihedral $\left(\delta_{\{\cdot\}}\right)$ and twist $\left(\theta_{\{\cdot\}}\right)$, respectively.Therefore,

$$
\begin{aligned}
& R_{1}=\left[\begin{array}{ccc}
\cos \beta_{R} & \sin \beta_{R} & 0 \\
-\sin \beta_{R} & \cos \beta_{R} & 0 \\
0 & 0 & 1
\end{array}\right], R_{2}=\left[\begin{array}{ccc}
1 & 0 & 0 \\
0 & \cos \delta_{R} & -\sin \delta_{R} \\
0 & \sin \delta_{R} & \cos \delta_{R}
\end{array}\right], \\
& R_{3}=\left[\begin{array}{ccc}
\cos \theta_{R} & 0 & -\sin \theta_{R} \\
0 & 1 & 0 \\
\sin \theta_{R} & 0 & \cos \theta_{R}
\end{array}\right] .
\end{aligned}
$$

Since the sweep angle is set to $\beta$. $\equiv 0$, we get the following rotation matrix which connects the right-wing root frame to the body frame:

$$
T_{\mathrm{BR}}=\left[\begin{array}{ccc}
1 & 0 & 0 \\
0 & \cos \delta_{R} & \sin \delta_{R} \\
0 & -\sin \delta_{R} & \cos \delta_{R}
\end{array}\right]\left[\begin{array}{ccc}
\cos \theta_{R} & 0 & \sin \theta_{R} \\
0 & 1 & 0 \\
-\sin \theta_{R} & 0 & \cos \theta_{R}
\end{array}\right] .
$$

A similar matrix $T_{B L}$ can be derived for the left wing as well.

\section{B. Equations of Motion}

In the following equations, given a vector $\mathbf{p}=\left[p_{1}, p_{2}, p_{3}\right]$,

$$
\mathbf{p} \times=S(\mathbf{p})=\left[\begin{array}{ccc}
0 & -p_{3} & p_{2} \\
p_{3} & 0 & -p_{1} \\
-p_{2} & p_{1} & 0
\end{array}\right]
$$

The translational equations are derived first. Let $\mathbf{r}_{c g}$ denote the position vector of the CG of the aircraft, while $\mathbf{r}_{c g, R}$ and $\mathbf{r}_{c g, L}$ denote the position vectors of the CG of the right and left wings, respectively. Then, the total translational momentum is given by

$$
\mathbf{p}=m \mathbf{u}_{B}+m\left(\omega_{B} \times \mathbf{r}_{c g}\right)+m_{w, R} \omega_{R} \times T_{B R} \mathbf{r}_{c g, R}+m_{w, L} \omega_{L} \times T_{B L} \mathbf{r}_{c g, L} .
$$

Using Newton's second law, we get

$$
\begin{aligned}
\mathbf{F}= & m\left(\dot{\mathbf{u}}_{B}+\omega_{B} \times \mathbf{u}_{B}+\dot{\omega}_{B} \times \mathbf{r}_{c g}+\omega_{B} \times \dot{\mathbf{r}}_{c g}+\left(\omega_{B} \times\right)^{2} \mathbf{r}_{c g}\right)+m_{w} \dot{\omega}_{R} \times T_{B R} \mathbf{r}_{c g, R} \\
& +m_{w}\left(\omega_{R} \times\right)^{2} T_{B R} \mathbf{r}_{c g, R}+m_{w} \dot{\omega}_{L} \times T_{B L} \mathbf{r}_{c g, L}+m_{w}\left(\omega_{L} \times\right)^{2} T_{B L} \mathbf{r}_{c g, L},
\end{aligned}
$$


and the $\mathrm{CG}$ variation is given by

$$
\dot{\mathbf{r}}_{c g}=\frac{m_{w}}{m}\left(\omega_{R} \times \mathbf{T}_{B R} r_{c g, R}+\omega_{L} \times T_{B L} \mathbf{r}_{c g, L}\right)
$$

The total angular momentum is given by

$$
\begin{aligned}
& \mathbf{h}=m \mathbf{r}_{c g} \times \mathbf{u}_{B}+J \omega_{B}+J_{R} \omega_{R}+J_{L} \omega_{L} \\
& J_{R}=T_{B R}^{\top} J_{R, R} T_{B R}, J_{L}=T_{B L}^{\top} J_{L, L} T_{B L}
\end{aligned}
$$

Therefore, the dynamical equations are given by

$$
\begin{aligned}
& \mathbf{M}=m \dot{\mathbf{r}}_{c g} \times \mathbf{u}_{B}+m \mathbf{r}_{c g} \times \dot{\mathbf{u}}_{B}+m \omega_{B} \times\left(\mathbf{r}_{c g} \times \mathbf{u}_{B}\right)+J \dot{\omega}_{B}+\dot{J} \omega_{B} \\
& +S\left(\omega_{B}\right) J \omega_{B}+J_{R} \dot{\omega}_{R}+\dot{J}_{R} \omega_{R}+S\left(\omega_{B}\right) J_{R} \omega_{R}+J_{L} \dot{\omega}_{L}+\dot{J}_{L} \omega_{L}+S\left(\omega_{B}\right) J_{L} \omega_{L},
\end{aligned}
$$

where

$$
\begin{aligned}
& \dot{J}_{R}=T_{B R}^{\top}\left(J_{R, R} S\left(\omega_{R}\right)-S\left(\omega_{R}\right) J_{R, R}\right) T_{B R}, \\
& \dot{J}=\dot{J}_{R}+\dot{J}_{L},
\end{aligned}
$$

and

$$
\mathbf{M}=\mathbf{M}_{\text {aero }}+\mathbf{r}_{c g} \times m\left[\begin{array}{c}
-g \sin \theta \\
g \cos \theta \sin \phi \\
g \cos \theta \cos \phi
\end{array}\right] .
$$

This paper studies the possible utility of differential dihedral and differential twist as control inputs. This entails that the aerodynamic forces and moments be described as functions of those angles and their rates of change. Strip theory is used to calculate the aerodynamic forces and the moments. Without any loss of generality, consider the right wing of an aircraft, with (semi) span $b / 2$ and chord $c(y)$, where $y$ denotes the spanwise location. Let $V_{c g}=\left[\begin{array}{lll}u & v & w\end{array}\right]^{\top}$ denote the body axis wind velocity of the aircraft. Let $\left[\begin{array}{ll}p & r\end{array}\right]$ denote the body axis angular velocity of the fuselage.

The angular velocity perceived at a strip at a distance $y$ along the span is independent of $y$ in a rigid seting, and is given by

$$
\boldsymbol{\Omega}=\left[\begin{array}{c}
p \\
q \\
r
\end{array}\right]+\left[\begin{array}{c}
0 \\
0 \\
\dot{\beta}_{R}
\end{array}\right]+R_{1}\left[\begin{array}{c}
-\dot{\delta}_{R} \\
0 \\
0
\end{array}\right]+R_{1} R_{2}\left[\begin{array}{c}
0 \\
\dot{\theta}_{R} \\
0
\end{array}\right]
$$

Then, the local velocity at that strip is

$$
\mathbf{V}(y)=\mathbf{V}_{c g}+\boldsymbol{\Omega} \times \mathbf{r},
$$

where $\mathbf{r}$ is the position vector of the aerodynamic center of the station given by

$$
\mathbf{r}=R_{1} R_{2} R_{3}\left[\begin{array}{c}
x_{a c} \\
y \\
0
\end{array}\right]
$$

where $x_{a c}$ is the chordwise location of the aerodynamic center with respect to the mid-chord. It is assumed to be $c / 4$. The local aerodynamic force at the station is given by the vector sum of the lift and the drag, with components written in the body frame:

$$
\mathbf{d} \mathbf{F}=0.5 \rho\|\mathbf{V}(y)\|^{2} c(y)\left(C_{l} \mathbf{l} /\|\mathbf{1}\|+C_{d} \mathbf{d} /\|\mathbf{d}\|\right) d y,
$$

where

$$
\begin{aligned}
\mathbf{l} & =R_{1} R_{2}\left[\begin{array}{lll}
0 & 1 & 0
\end{array}\right]^{\top} \times \mathbf{V}(y) /\|\mathbf{V}(y)\|, \\
\mathbf{d} & =-\mathbf{V}(y) /\|\mathbf{V}(y)\| .
\end{aligned}
$$


The local aerodynamic moment at the station is given by

$$
\mathbf{d} \mathbf{M}=\mathbf{r} \times \mathbf{d} \mathbf{F}+\rho\|\mathbf{V}(y)\|^{2} c(y) C_{m_{a c}} R_{1} R_{2}\left[\begin{array}{lll}
0 & 1 & 0
\end{array}\right]^{\top} d y
$$

The total aerodynamic force and moment are obtained by integrating the above expressions, performed in practice by using a finite element scheme. The forces can be projected onto the wind axes and these, together with the moments, can be substituted into the flight dynamic equations.

In addition to the flight dynamic equations, we have the kinematic equations which relate the angular velocity of the aircraft to the rates of change of the Euler angles:

$$
\begin{aligned}
\dot{\phi} & =p+q \sin \phi \tan \theta+r \cos \phi \tan \theta \\
\dot{\theta} & =q \cos \phi-r \sin \phi \\
\dot{\psi} & =(q \sin \phi+r \cos \phi) / \cos \theta .
\end{aligned}
$$

The equations which relate the position of the aircraft to its translational velocity are essentially decoupled from the flight dynamic equations, and are given by

$$
\begin{aligned}
\dot{X} & =V \cos \gamma \cos \chi \\
\dot{Y} & =V \cos \gamma \sin \chi \\
\dot{Z} & =-V \sin \gamma .
\end{aligned}
$$

The angles $\gamma$ and $\chi$ in Eq. (21) are defined as follows:

$$
\begin{aligned}
\sin \gamma= & \cos \alpha \cos \beta \sin \theta-\sin \beta \sin \phi \cos \theta-\sin \alpha \cos \beta \cos \phi \cos \theta \\
\sin \chi \cos \gamma= & \cos \alpha \cos \beta \cos \theta \sin \psi+\sin \beta(\sin \phi \sin \theta \sin \psi+\cos \phi \cos \psi) \\
& +\sin \alpha \cos \beta(\cos \phi \sin \theta \sin \psi-\sin \phi \cos \psi)
\end{aligned}
$$

The aerodynamic data from [13] used here has been verified for high angles of attack. The lift and drag coefficients of the wing and tail airfoils are

$$
C_{l}=0.225+1.58 \sin (2.13 \alpha-7.20), C_{d}=1.92-1.55 \cos (2.04 \alpha-9.82),
$$

where $\alpha$ is measured in degrees. The aircraft geometry is as follows:

- Wing span: $37.5 \mathrm{~cm}$, chord: $14.6 \mathrm{~cm}$.

- Horizontal tail span: $19 \mathrm{~cm}$, chord: $9 \mathrm{~cm}$.

- Mass: 12 g., including a ballast mass added to the nose of the aircraft for placing the CG around half-wing-chord under nominal conditions (i.e., when the wing dihedral and twist set to zero).

- Length: $29.7 \mathrm{~cm}, x_{a c}=3.6 \mathrm{~cm}, x_{c g}=5.5 \mathrm{~cm}$ (from the nose when $\delta_{R}=\delta_{L}=\delta_{e}=0$ ).

\section{Discussion}

\section{A. Analytical Comparison With the Vertical Tail}

Figure 5 illustrates the physics underlying the use of wing dihedral as a control. Increasing the wing dihedral reduces the force acting in the $z$ - direction, and generates a side force. The reduced $z$-force affects the aircraft flight path angle and angle of attack, and hence the flight speed. On the other hand, the side force can be used for providing the centripetal force for turning, and as a source of the yawing moment. In particular, if the CG is located behind the line of action of the side force, then a positive side force produces a positive yawing moment and vice-versa. This means that a positive rolling moment (wherein the lift on the left wing is higher than the right wing) is accompanied by a positive yawing moment if the wings have a positive dihedral deflection or if the dihedral deflection of the left wing is larger than that of the right wing. This helps reduce the adverse yaw produced due to rolling.

In order to appreciate the utility and the limitations of using differential dihedral as yaw control mechanism, it may be compared it with the more conventional vertical tail by comparing the yawing moments 


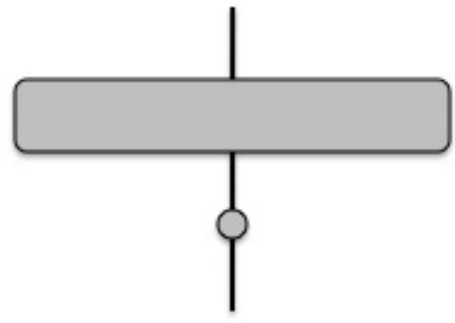

Wing ahead of CG

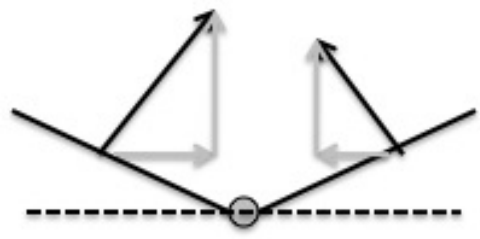

Excess lift on one wing produces side force and yawing moment

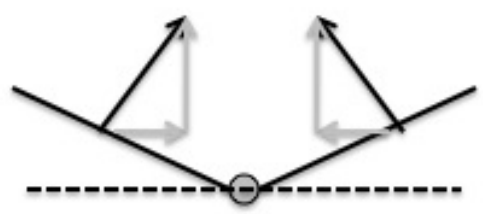

Reduce net lift without altering drag

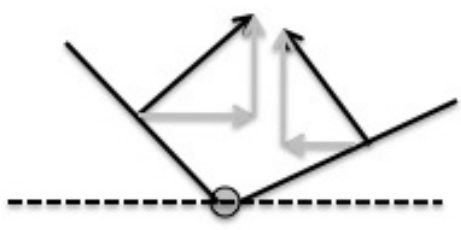

\section{Larger dihedral on one wing produces side force and yawing moment}

Figure 5. Illustration of the physics underlying the use of dihedral as a control.

produced by the differential dihedral and the vertical tail while their deflections are kept identical. Consider a rectangular vertical tail with span $b_{t}$, chord $c_{t}$, area $S_{t}=b_{t} c_{t}$, and located at a distance $l_{t}$ behind the CG. The wing has semi-span $b / 2$ and chord $c$. Let the $S_{w}=b c / 2$ denote the area of each wing, and let the wing be located at a distance $l_{w}$ ahead of the CG. In order to get a fair comparison, the yawing moment produced by the vertical tail is calculated by assuming that the dihedral deflection of both wings is zero.

Assuming a linear relation between the lift and the angle of attack, the yawing moment generated by the tail for a deflection $\beta_{t}$ is equal to $M_{t}=q_{\infty} S_{t} l_{t} C_{L_{\alpha}} \beta_{t}$, where $q_{\infty}$ is the free stream dynamic pressure. The moment generated by a dihedral deflection, $\delta$, of one of the wings is $M_{w}=q_{\infty} S_{w} l_{w} C_{L_{\alpha}} \alpha_{w} \delta$. If $\beta_{t}=\delta$, then the ratio $e=M_{w} / M_{t}$ is given by

$$
e=\frac{S_{w} l_{w}}{S_{t} l_{t}} \alpha_{w}
$$

Clearly, the dihedral is more effective as a control at high angles of attack. Furthemore, the deterioration in the moment generated by the tail when it lies in the wing wake has been ignored in the above calculations. Accounting for this deterioration will improve $e$ further. The above expression also suggests that the dihedral is better than the vertical tail when the aircraft has a small tail arm. Interestingly enough, blended wing body aircraft and birds are candidates for such aircraft where differential dihedral offers a more efficient option for yaw control. This is one of the reasons, apart from the stability arguments of Sachs, ${ }^{10}$ why birds can do away with the vertical tail altogether. The ability to change wing dihedral is built into the birds in the form of their ability to flap their wings for propulsion. Hence, no additional mechanisms are needed for yaw control. Ornithopters, too, can benefit from differential dihedral-based yaw control in a similar manner.

\section{B. Longitudinal Stability and Performance}

This subsection illustrates two points:

- The use of bifurcation analysis for trim and stability analysis, and

- The effect of dihedral on the longitudinal trim performance. 
It has been assumed that the wing twist and sweep are both zero.

Figure 6 is a bifurcation diagram of $\alpha$ versus $\delta_{e}$ for longitudinal flight with $\delta_{R}=\delta_{L}=0.5$ rad. The trim angle of attack is shown on the vertical axis, while the corresponding elevator deflection is shown on the x-axis. The following notation is adopted for the plots: filled circles indicate stable equilibria, while other markers are used for unstable equilibria depending on the type of instability. The aircraft is seen to be dynamically stable at all angles of attack considered in this plot. Such a study can be performed for different values of the dihedral angle to ascertain the safe flying envelope.

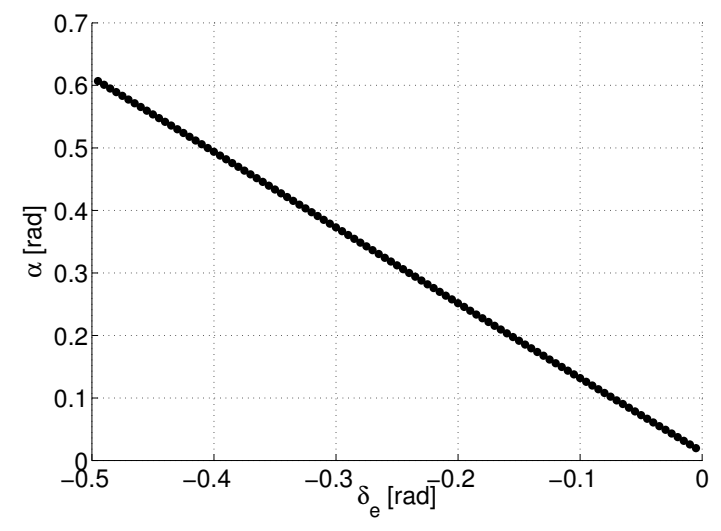

Figure 6. Bifurcation diagram showing $\alpha$ versus $\delta_{e}$ for $\delta_{R}=\delta_{L}=0.5 \mathrm{rad}$.

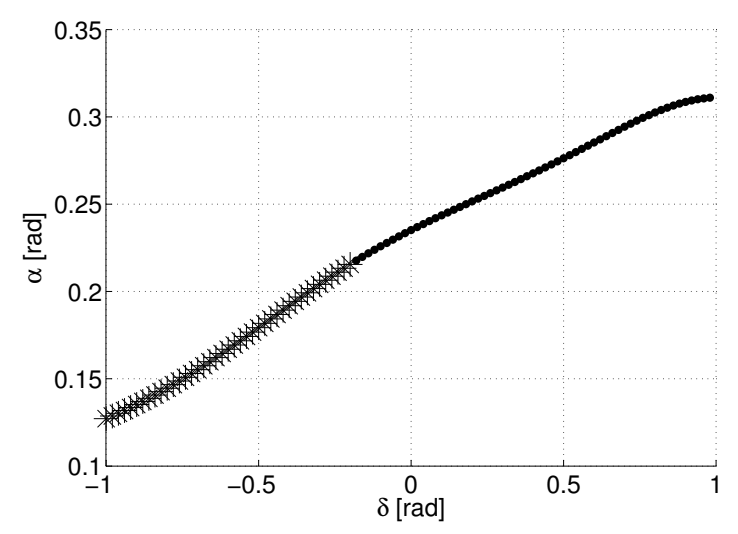

(a) Angle of attack as a function of dihedral.

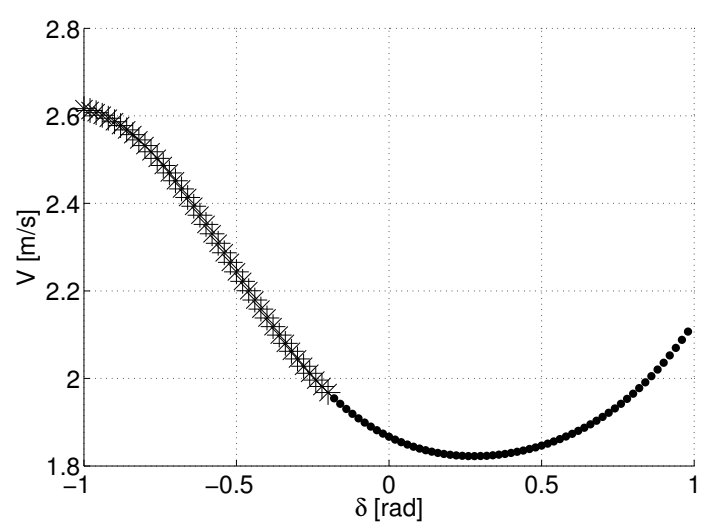

(b) Velocity as a function of the dihedral

Figure 7. Bifurcation diagrams showing $\alpha$ and flight speed versus symmetric dihedral (i.e., $\delta_{R}=\delta_{L}=\delta$ ) with elevator held fixed at $\delta_{e}=-0.2 \mathrm{rad}$. Solid circles (.) indicate stable equilibria, while a cross (' $\mathrm{x}$ ') indicates unstable equilibria with one real unstable eigenvalue.

In order to study the effect of the wing dihedral on the longitudinal performance and stability, the elevator deflection is kept fixed. The dihedral angle is varied. Changing the wing dihedral changes the trim angle of attack and trim velocity substantially as shown in Figs. 7(a) and 7(b). In particular, increasing the wing dihedral increases the trim angle of attack. As the dihedral is increased, the speed reaches a minimum value around $\delta=0.25 \mathrm{rad}$ before increasing for higher values of the dihedral. The equilibria are unstable for dihedral values below $-0.2 \mathrm{rad}$.

The key observation here is that the use of symmetric dihedral offers the possibility of independently controlling two of the three longitudinal performance metrics, viz. the flight speed, angle of attack and the flight path angle. The Euler pitch angle would be an enterprising addition to this list, with application to airborne visual tracking of other airborne vehicles or ground-based objects. This is not possible in fixed wing aircraft in the absence of variable thrust and variable dihedral. Figure 8(a) shows the angle of attack required 
to maintain a flight speed of $V=2.2 \mathrm{~m} / \mathrm{s}$ as the symmetric dihedral is varied between -1.0 and $1.0 \mathrm{rad}$, while Fig. 8(b) shows the flight path angle achieved in the process. The elevator needs to be scheduled as a function of the dihedral deflection for steady flight, as shown in Fig. 9. In the last three plots, the equilibria are seen to be unstable with a single positive real eigenvalue for large negative values of the wing dihedral.

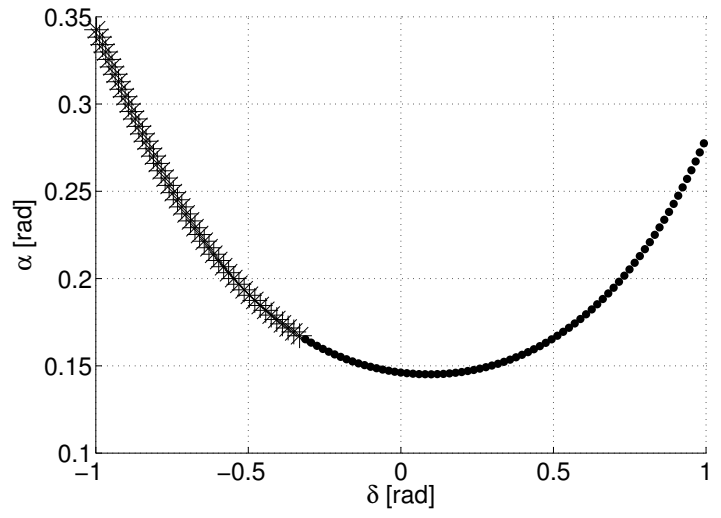

(a) Angle of attack as a function of dihedral.

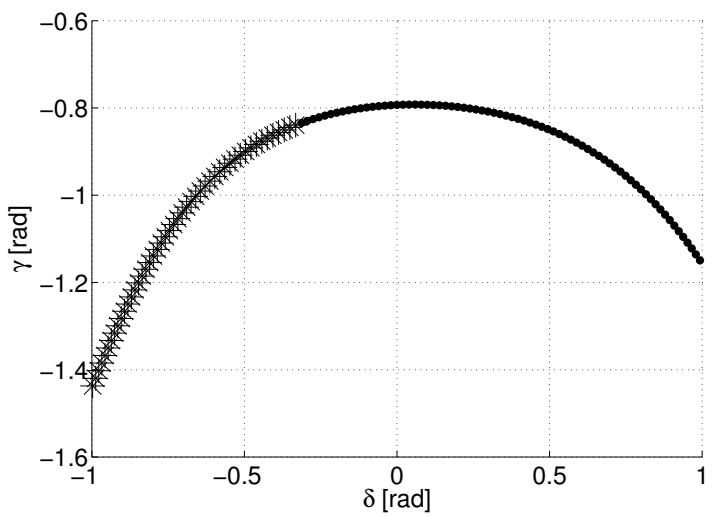

(b) Flight path angle as a function of the dihedral

Figure 8. Bifurcation diagrams showing $\alpha$ and $\gamma$ versus symmetric dihedral (i.e., $\delta_{R}=\delta_{L}=\delta$ ) with speed held fixed at $V=2.2 \mathrm{~m} / \mathrm{s}$. Solid circles (.) indicate stable equilibria, while a cross (' $\mathrm{x}$ ') indicates unstable equilibria with one real unstable eigenvalue.

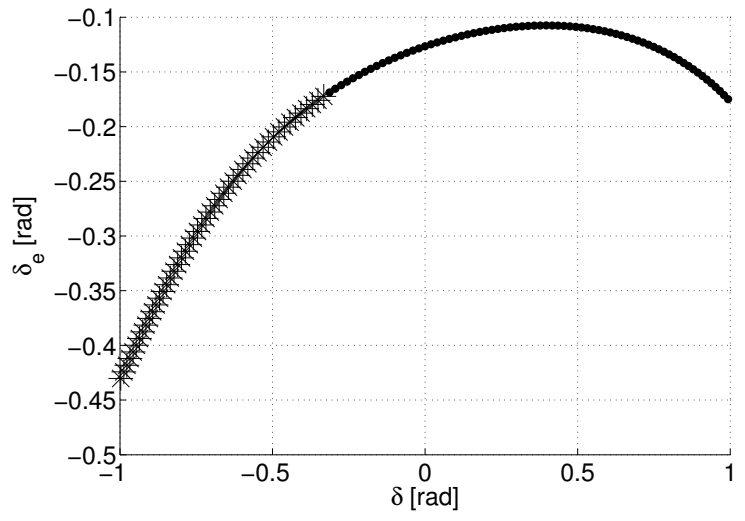

Figure 9. Bifurcation plot showing the elevator required to maintain the flight speed as the symmetric dihedral deflection, $\delta$, is varied. Solid circles $(\cdot)$ indicate stable equilibria, while a cross (' $x$ ') indicates unstable equilibria with one real unstable eigenvalue.

The steady state analysis demonstrated here is of limited utility insofar as determining the agility of the aircraft is concerned, because agility is evaluated primarily on the basis of transient maneuvers such as perching [1]. Nevertheless, a steady state analysis is useful for two reasons. First, as stated earlier, it helps identify potential instabilities. Second, an aircraft with well damped dynamics would tend to rapidly approach the steady state velocity and angle of attack predicted here, even as it performs an unsteady maneuver.

\section{Lateral Stability and Performance}

Wing twist can be used anti-symmetrically on the two wings to generate rolling moment, and differential dihedral can serve as a yaw control mechanism as explained in subsection A. Roll rate and turn rate are two indicators of lateral performance. As in the previous section, bifurcation analysis is used for analysing performance and stability for following benchmark cases: 
1. Dihedral angle on both wings is equal and held constant ( $0.1 \mathrm{rad})$ as twist is varied in an antisymmetric manner. It is expected that this will generate a substantial roll rate as well as sideslip.

2. The wing twist is varied as in the previous case. However, the dihedral angles are not held constant. Instead, they are scheduled to ensure zero sideslip and constant flight speed. In this case, the aircraft performs a steady coordinated turn.

Figures 10(a) and 10(b) show the roll rate and the sideslip angle as functions of the twist angle for constant dihedral angle. The ideas discussed above are readily verified. It is seen that a large roll rate is generated for relatively small values of anti-symmetric wing twist. This may explain the roll agility seen in birds. In order

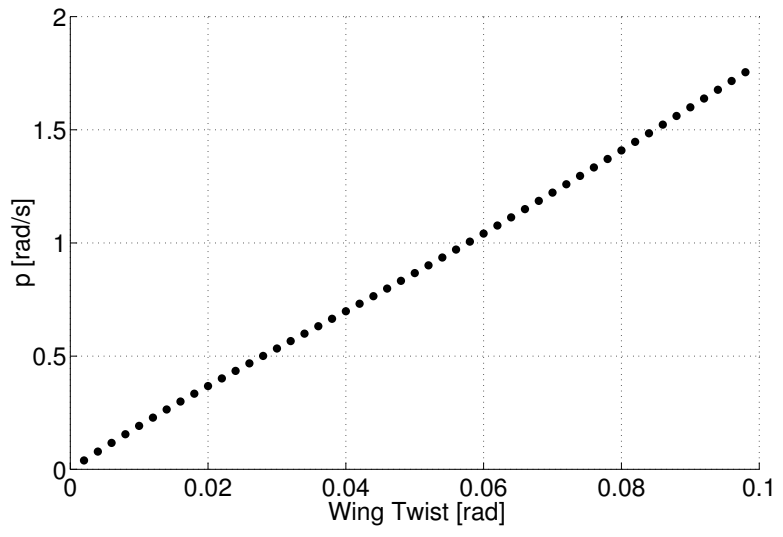

(a) Roll rate as a function of antisymmetric wing twist (left wing positive)

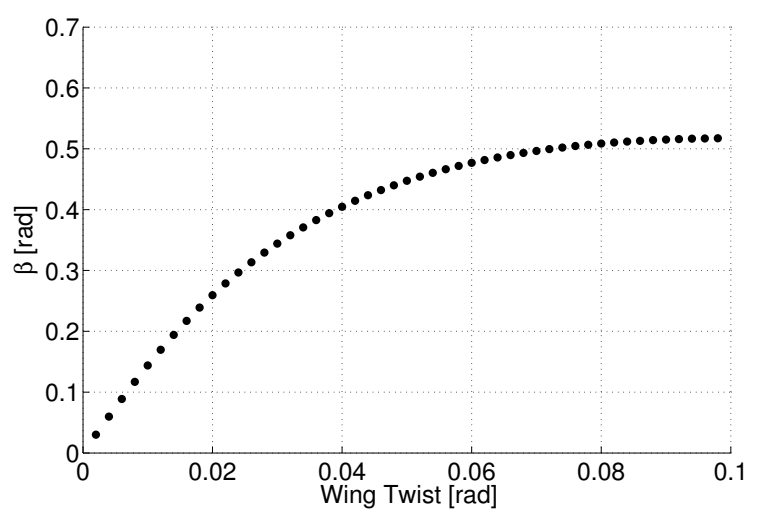

(b) Sideslip as a function of antisymmetric wing twist

Figure 10. Roll rate and sideslip as functions of wing twist.

to analyse a turning maneuver, a simplified version of constrained bifurcation analysis (CBA)[14] is employed. In CBA, constraint equations are added to the ordinary differential equations and the corresponding control parameters are "freed," i.e., treated as state variables. The enlarged set of equations is then solved to compute the aircraft trim states and control inputs needed to achieve those trims. In order to conclude the stability of the trim states, the Jacobian corresponding only to the original set of differential equations is isolated, and its eigenvalues are computed.

Figures 11(a) and 11(b) plot the turn rate and the differential dihedral required for regulating sideslip, respectively, as functions of the wing twist during a coordinated turn. From a performance perspective, it is observed that a substantial amount of asymmetric (not necessarily anti-symmetric) dihedral is needed to maintain zero sideslip. During this maneuver, the speed is held constant at $V=2 \mathrm{~m} / \mathrm{s}$. From the stability perspective, the maneuver is seen to be stable. However, instabilities may appear in steep turns as observed, for example, in Paranjape and Ananthkrishnan [16].

Figure 11(b) also shows that $\delta_{R} \approx-\delta_{L}$ for trim at small values of wing twist. This is equivalent to an aircraft banking to one side while performing a turn. This is observed for small values of turn rate, upto $10 \mathrm{deg} / \mathrm{s}$. The corresponding pitch rate and fuselage bank angle are both small. As the turn rate builds up, so does the pitch rate as the aircraft bank angle increases, which leads to a deviation from this simple expression. Even so, the aircraft bank angle (not shown here) does not exceed 10 deg even for rapid turns.

\section{Simulations}

The previous sections demonstrated the steady state performance of an MAV equipped with variable wing dihedral and twist. Agile maneuvers, on the other hand, are purely transient in nature. The ability of the aircraft to perform such maneuvers can be demonstrated only by simulation. In this section, a perching maneuver is demonstrated as an illustration of the aircraft's agility. For the purpose of simulations, a simple PI controller is implemented wherein the angle of attack is fed back to the wing twist as well as the elevator deflection. The dihedral angles are commanded in the open loop. 


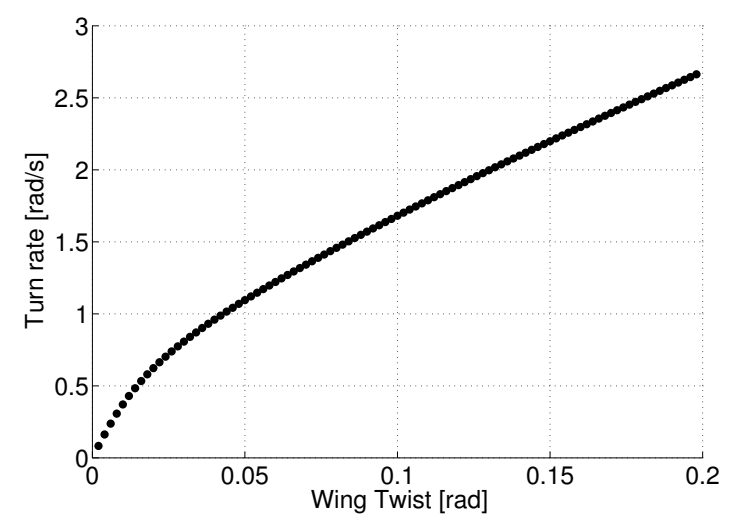

(a) Turn rate and sideslip as functions of wing twist with constraint $\beta=0$.

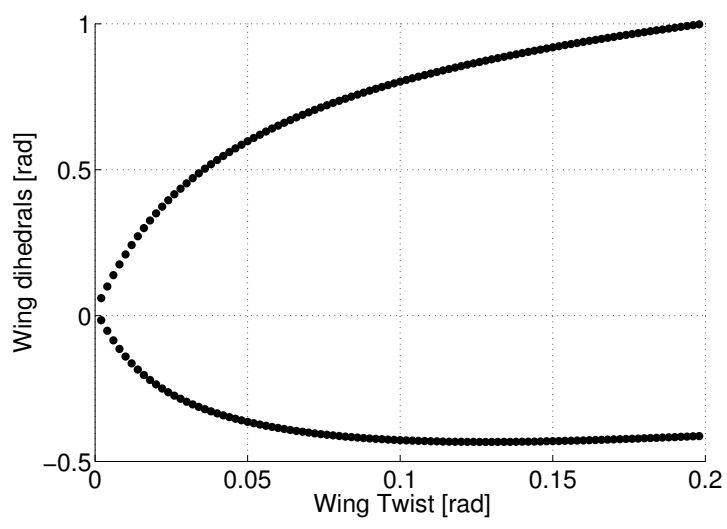

(b) Wing dihedral required to maintain $\beta=0$.

Figure 11. Coordinated turn trims with a fixed velocity $V=2 \mathrm{~m} / \mathrm{s}$.

A simple perching maneuver is envisioned as follows. The aircraft descends steeply, at a moderate angle of attack. The dihedral is initally set to $0.5 \mathrm{rad}$. At $t=4 \mathrm{~s}$., the dihedral is reduced to 0 , and the wings are twisted upwards so that the aircraft pitches up instantaneously and the speed drops rapidly. Perching would be ideally completed when the speed has dropped to its minimum value. The flight speed, angle of attack and flight path angle have been plotted in Figs. 12(a) and 12(b), respectively. It is seen that the flight path angle becomes positive instantaneously. This is in keeping with the perching maneuver described in literature, for example [1]. Figure 12(c) shows the wing twist. The wing twist is scheduled so that the wing angle of attack roughly equals $0.4 \mathrm{rad}$. Exact equality is not always possible due to saturation limits on wing twist. The perching trajectory has been shown in Fig. 12(d). Incidentally, the first part of the maneuver can be used for performing rapid descents in constrained space, as a substitute or complement for strategies such as the spin-based strategy discussed by Ramsey and co-authors [17]. Chakravarthy and co-authors ${ }^{18}$ have designed a guidance algorithm based on $\mathcal{H}_{2}$ optimal control for perching using the ideas described in this paper.

\section{Experimental Results}

The ideas presented in this paper are being validated experimentally. In this section, some results obtained during open loop studies have been presented. Experiments were performed on Plantraco's Kolibri Pocket Plane (see Figs. 2(a) and 2(b)). The aircraft has a wing span of $220 \mathrm{~mm}$, and weighs approximately $5 \mathrm{~g}$. The aircraft wings were modified so that their dihedral could be changed a priori. Ailerons were added as substitutes for wing twist, and the vertical tail was duly removed. The aircraft was seen to possess a significantly large $L / D$ ratio. Ballast mass was added to the aircraft to rein in its tendency to accelerate and pull up. Spoilers were added on the inboard section of the wing to improve phugoid damping. Experiments were performed with the elevator set to $\delta_{e}=-10 \mathrm{deg}$. The aircraft position was measured directly using VICON and the sampling frequency was set to $100 \mathrm{~Hz}$. The velocities and the heading angles were derived from the position measurements using the finite difference method. For longitudinal studies, the angle of attack was approximated as the difference between the body pitch angle and the flight path angle. Even with the added mass and spoilers, the phugoid motion did not damp out sufficiently within the available experimental space for small values of the wing dihedral. Therefore, the mean values of the flight parameters, as the expected steady state values, were noted down.

\section{A. Longitudinal}

Figures 13(a) - 13(c) show the flight speed, angle of attack and flight path angle as a function of the wing dihedral. Flight tests at small values of the dihedral were precarious, because of the tendency of the aircraft 


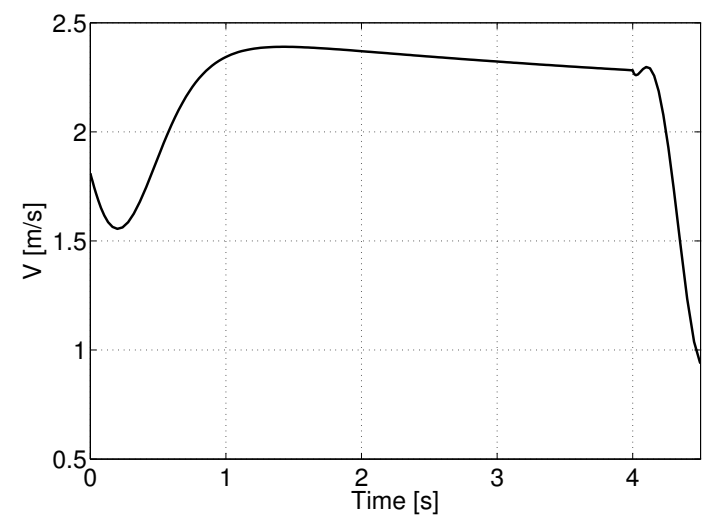

(a) Flight speed as a function of time.

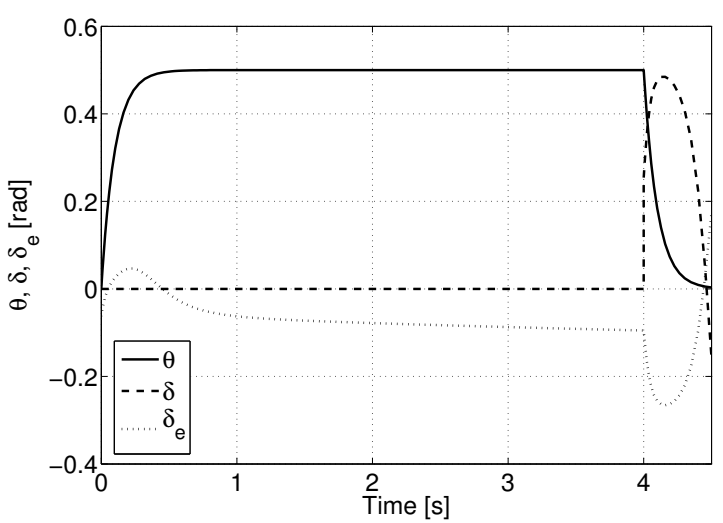

(c) Symmetric wing twist (solid), symmetric dihedral angle (dashed), and elevator (finely dashed) time histories during perching.

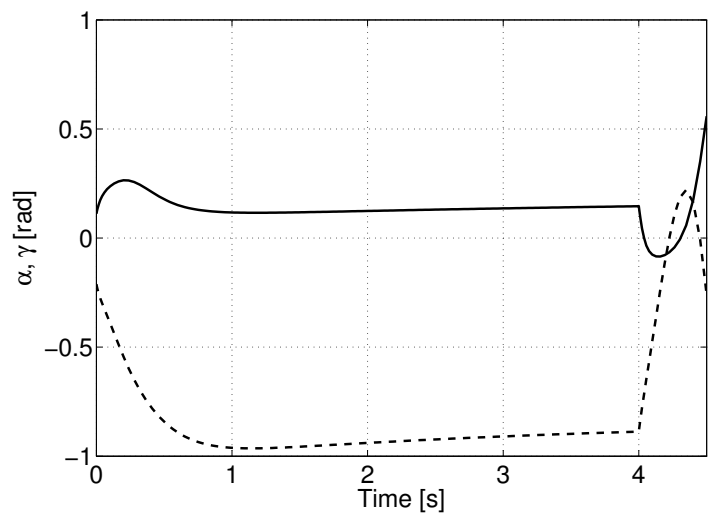

(b) Angle of attack (solid line) and flight path angle (dashed line) time histories.

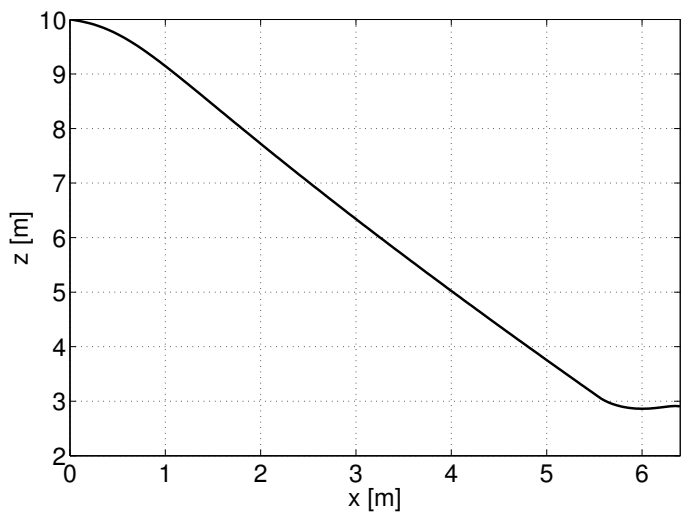

(d) Perching trajectory in the $x-z$ plane.

Figure 12. Flight parameters during a perching-like maneuver.

to depart into a spiral. Nevertheless, the velocity and the flight path angle trends (also shown by a quadratic fit) match those predicted by the theoretical analysis in the preceding section. The angle of attack decreases almost linearly as the dihedral angle is decreased from 55 deg to 20 deg. The angle of attack behaviour is seen to deviate substantially thereafter, and it may be partially attributed to the truant dynamics of the aircraft. Consequently, no attempt was made to fit a lower order curve to the angle of attack data.

\section{B. Lateral}

Attempts were made to study the turning performance of the aircraft for different values of the aileron deflection. The aircraft, however, was seen to be unstable with problems similar to those faced with the longitudinal experiments. Whereas the latter could be circumvented by adding mass and drag, the problems with lateral stability could not be adequately countered. Some of the results have been tabulated in Table 1. The aileron deflection has been denoted by $\delta_{a}$. The aircraft was seen to possess a very poorly damped transient performance. Consequently, the values in Table 1 are approximate, in that they are obtained as the expected steady state solutions. Beyond $\delta_{a}=-15 \mathrm{deg}$, the aircraft became unstable ruling out any meaningful turning experiments in the open loop. The data presented in Table 1 is, nevertheless, instructive in its own right. 


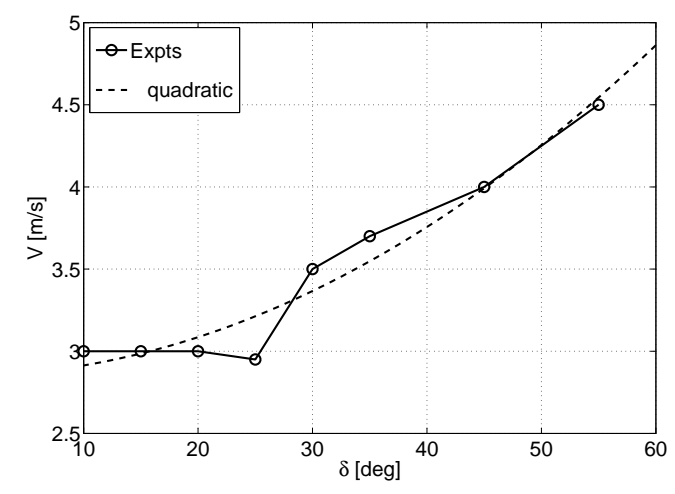

(a) Flight speed as a function of the dihedral.

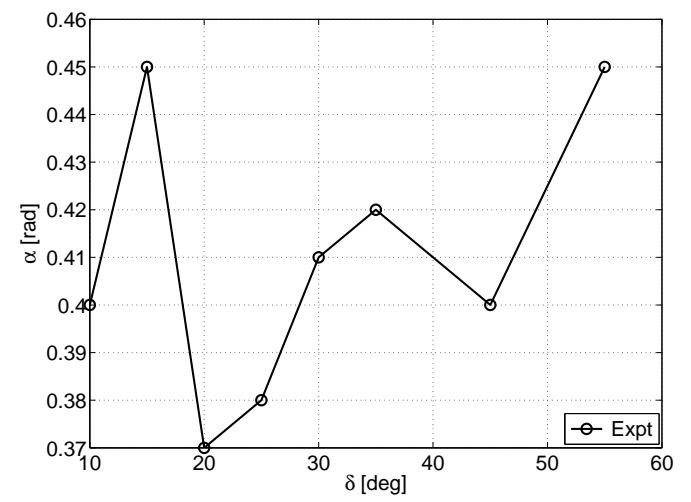

(b) Angle of attack as a function of the dihedral.

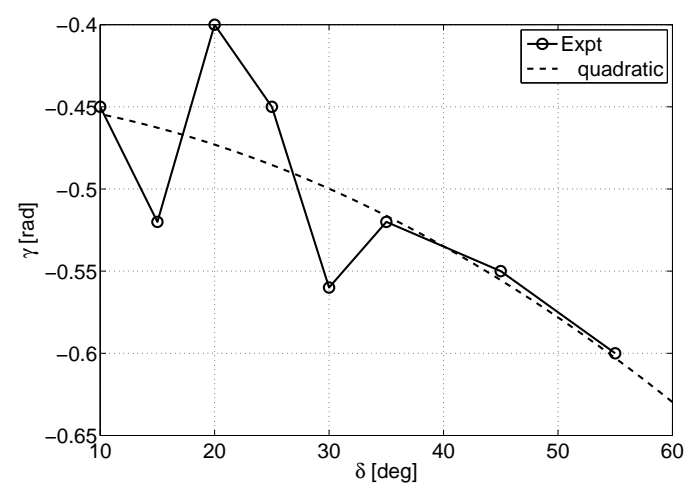

(c) Flight path angle as a function of the wing dihedral.

Figure 13. Steady state values of the longitudinal flight parameters as functions of the wing dihedral.

For $\delta_{a}=-5 \mathrm{deg}$, note that the aircraft sideslip increases as $\delta_{R}-\delta_{L}$ is increased. The desired coordinated turn would hence occur when $\delta_{L}$ is just less than $5 \mathrm{deg}$. The flight speed is almost constant, which matches the expectations from the earlier trim analysis. The aircraft turn rate increases as $\delta_{R}-\delta_{L}$ increases due of the consequent increase in the side force.

For $\delta_{a}=-10 \mathrm{rad}$, the sideslip is zero when $\delta_{L}=-10 \mathrm{deg}$, and $\delta_{R}$ is between 10 and $15 \mathrm{deg}$. Note that the turn rate and flight speed are almost constant. Therefore, a part of the increased sideforce is lost in compensating for the sideslip. It is interesting that even such sparse and crude, albeit carefully chosen, data can be useful for predicting the optimal dihedral combination for coordinated turns.

\section{Conclusions}

The ultimate goal of this work is to develop agile MAVs that can mimic or exceed the agile maneuvers of birds. This paper demonstrates the effect of wing dihedral on aircraft performance and stability. Wing dihedral, together with the horizontal tail, can be used to control the aircraft angle of attack (or flight path angle) and flight speed independently for a range of flight speeds. Asymmetric dihedral deflection has been demonstrated as a useful yaw control mechanism for agile MAVs. Future work on this front should focus on (a) the development of aggressive control laws which incorporate asymmetric dihedral actuation, and (b) the effect of wing flexibility, particularly torsion, given the sensitivity of the roll rate to asymmetric wing twist. Both problems are currently under investigation by the authors. 


\begin{tabular}{cccccc}
\hline$\delta_{a}(\mathrm{deg})$ & $\delta_{R}(\mathrm{deg})$ & $\delta_{L}(\mathrm{deg})$ & $V(\mathrm{~m} / \mathrm{s})$ & $\omega(\mathrm{rad} / \mathrm{s})$ & $\beta(\mathrm{rad})$ \\
\hline \hline-5 & 5 & -5 & 3.6 & 2 & 0.08 \\
& 10 & -5 & 3.74 & 2.33 & 0.14 \\
& 10 & -10 & 3.8 & 2.7 & 0.23 \\
\hline-10 & 10 & -10 & 3.5 & 2.8 & -0.08 \\
& 15 & -10 & 3.5 & 2.8 & 0.2 \\
\hline
\end{tabular}

Table 1. A summary of the turning performance. The aileron deflection has been denoted by $\delta_{a}$. The negative sign implies that the aircraft rolls to the left.

\section{Acknowledgments}

This project was supported by the Air Force Office of Scientific Research (AFOSR) under the Young Investigator Award Program (Grant No. FA95500910089) monitored by Dr. W. Larkin. The original problem was posed by Dr. Gregg Abate (AFRL). This paper also benefitted from stimulating discussions with Mr. Johnny Evers (AFRL). Dr. Animesh Chakravarthy (Univ. of Florida REEF) provided useful feedback and comments from the very inception of this work, and helped refine the paper to the present state. The authors also thank undergraduate research assistants James Holtman, Joe Kim, Andrew Meister, Daniel Park and Jonathon Yong whose hard work resulted in an experimental MAV for evaluating the ideas described in this paper.

\section{References}

${ }^{1}$ Ol, M., Parker, G., Abate, G., and Evers, J., "Flight Controls and Performance Challenges for MAVs in Complex Environments," AIAA Guidance, Navigation and Control Conference and Exhibit, 2008. AIAA Paper 2008 - 6508.

${ }^{2}$ Paranjape, A. A., Chakravarthy, A., Chung, S.-J., and Hilton, H. H., "Performance and Stability of an Agile MAV with Flexible Articulated Wings," Proc. AIAA Atmospheric Flight Mechanics (AFM) Conference, Toronto, Canada, 2010.

${ }^{3}$ Mueller, T. J., Fixed and Flapping Wing Aerodynamics for Micro Air Vehicle Applications, Progress in Aeronautics and Astronautics, Vol. 195, AIAA, 2001.

${ }^{4}$ Costello, M., and Webb, A., "Wing Articulation of Micro Air Vehicles to Reduce Gust Sensitivity," AIAA Paper 2008 6712,2008

${ }^{5}$ Wickenheiser, A., and Garcia, E., "Longitudinal Dynamics of a Perching Aircraft," Journal of Aircraft Vol. 43, No. 5, 2006, pp. $1386-1392$.

${ }^{6}$ Wickenheiser, A., and Garcia, E., "Optimization of Perching Maneuvers through Vehicle Morphing," Journal of Guidance, Control and Dynamics, Vol. 31, No. 4, 2008, pp. 815 - 823.

${ }^{7}$ Reich, G. W., Wojnar, O., and Albertani, R., "Aerodynamic Performance of a Notional Perching MAV Design," AIAA Paper 2009 - 63, 2009.

${ }^{8}$ Sachs, G., and Holzapfel, F., "Flight Mechanic and Aerodynamic Aspects of Extremely Large Dihedral in Birds," AIAA Paper 2007 - 46, 2007.

${ }^{9}$ Sachs, G., "What Can Be Learned From Unique Lateral-Directional Dynamics Properties of Birds for Mini-Aircraft," AIAA 2007-6311, 2007.

${ }^{10}$ Sachs, G., "Why Birds and Miniscale Airplanes Need No Vertical Tail," Journal of Aircraft, Vol. 44, No. 4, 2007, pp. 1159 $-1167$.

${ }^{11}$ Paranjape, A. A., and Chung, S.-J., "Lateral Stability of Vertical-Tailless Aircraft with Variable Wing Dihedral," under review, Journal of Guidance, Control and Dynamics, 2010."

${ }^{12}$ Chung, S.-J., and Dorothy, M., "Neurobiologically Inspired Control of Engineered Flapping Flight," Journal of Guidance, Control and Dynamics, Vol. 33, No. 2, 2010, pp. 440 - 453.

${ }^{13}$ Dickinson, M. H., Lehmann, F. -O., and Sane, S. P., "Wing Rotation and the Aerodynamic Basis of Insect Flight," Science, Vol. 284, 1999, pp. 1954 - 1960.

${ }^{14}$ Paranjape, A. A., Sinha, N. K., and Ananthkrishnan, N., "Use of Bifurcation and Continuation Methods for Aircraft Trim and Stability Analysis - A State-of-the-Art" Journal of Aerospace Sciences and Technologies, Vol. 60, No. 2, 2008, pp. 1-12. Abridged version: AIAA Paper 2007 - 1051.

${ }^{15}$ Keller, H. B., "Numerical Solution of Bifurcation and Nonlinear Eigenvalue Problems," in Applications of Bifurcation Theory, P. Rabinowitz Ed., Academic Press, 1977.

${ }^{16}$ Paranjape, A. A., and Ananthkrishnan, N., "Aircraft Level Turn Performance, Including Stability Constraints, Using EBAC Method" AIAA Paper 2005 - 5898, 2005.

${ }^{17}$ Ramsey, J. A., Ratliff, R. T., Wise, K. A., and Lavretsky, E., "Departure Resilient Control for Autonomous Air Vehicles," Proc. 2009 American Control Conference, 2009, pp. 5386 - 5391.

${ }^{18}$ Chakravarthy, A., Paranjape, A. A., and Chung, S.-J., "Control Law Design for Perching an Agile MAV with Articulated Wings," Proc. AIAA Atmospheric Flight Mechanics (AFM) Conference, Toronto, Canada, 2010. 